\title{
MONITORING PROTON THERAPY THROUGH IN-BEAM PET: AN EXPERIMENTAL PHANTOM STUDY
}

\author{
A. Topi, S. Muraro, G. Battistoni, N. Belcari, M.G. Bisogni, N. Camarlinghi, A. Del Guerra, A. Ferrari, R. \\ Kopec, A.C. Kraan, D. Krzempek, K. Krzempek, M. Morrocchi, P. Olko, P. Sala, G. Sportelli and V. Rosso
}

\begin{abstract}
In this work, we investigate the use of a PET system to monitor the proton therapy. The monitoring procedure is based on the comparison between the $\beta+$ activity generated in the irradiated volume during the treatment, with the $\beta$ +activity distribution obtained with Monte Carlo simulation.

The dedicated PET system is a dual head detection system; each head is composed of nine scintillating LYSO crystal matrices read out independently with a custom modularized acquisition system.

Our experimental data were acquired at the Cyclotron Centre Bronowice, Institute Nuclear Physics in Krakow, Poland and were simulated with the FLUKA Monte Carlo code. Homogeneous and heterogeneous plastic phantoms were irradiated with monoenergetic $130-\mathrm{MeV}$ protons. The capabilities of our PET system to distinguish different irradiated materials were investigated, and the proton pencil beams were used as probes. Our focus was to analyze the activity width and the total activity event number in several cases. Irradiations were performed using either single pencil beams one at a time, or two pencil beams during the same data taking. The comparison of 1D activity profile for experimental data and MC simulation were always in good agreement showing that, the treatment quality assessment in proton therapy can be based on $\beta+$ activity measurements.
\end{abstract}

Index Terms-Proton Therapy, Positron Emission Tomography, Treatment Monitoring, Monte Carlo

\section{INTRODUCTION}

$\mathrm{P}$ roton therapy allows to deliver dose to the tumor in a highly conformal way, thanks to the sharp dose profile (Bragg peak). Treatments with protons are more sensitive to uncertainties than photon treatments, because small variations in proton range can result in large variations in delivered dose. This often leads to the usage of large safety margins and highly conservative treatment plans. The full potential of

A. Topi is with Istituto Nazionale di Fisica Nucleare, Sezione di Pisa, Italy, and with Department of Physical Sciences, Earth and Environment, University of Siena, Italy.

S. Muraro and A.K. Kraan are with Istituto Nazionale di Fisica Nucleare, Sezione di Pisa, Italy.

G. Battistoni and P. Sala are with Istituto Nazionale di Fisica Nucleare, Sezione di Milano, Italy. proton therapy is thus not yet fully exploited [1]. For this reason, various techniques to monitor the treatment quality invivo have been developed in the last decades [2, 3].

Among these techniques, there is proton range monitoring with Positron Emission Tomography (PET). Based on the acquisition of back to back photons from the $\beta+$ annihilation, it is possible to reconstruct the position of the $\beta+$ emitters produced by proton interactions with the patient tissue during irradiation. Different data acquisition modalities are off-beam, in-beam and in-room PET [4-14]. PET monitoring is conventionally done by acquiring data during or after irradiation, and comparing the data with reference distributions, either from Monte Carlo (MC) or from previous treatment sessions $[15,18]$. This allows extracting valuable information about the correctness of the treatment a posteriori. In view of the growing interest in image guidance procedures and dose escalation, it is interesting to investigate the use of PET as pre-treatment check using just a few selected pencil beams, in order to provide information about patient positioning problems, morphological changes etc. Although acquiring sufficient statistics is a major challenge, in-beam PET monitoring at short times could be one of the possible candidates for this purpose. PET monitoring based on a few single pencil-beams would have an additional advantage for the MC simulations, because it would strongly reduce the calculation time and complexity of the MC calculations with respect to simulating a complete treatment plan [19].

In this work, we present a feasibility study that shows how an in-beam PET system can be used for the treatment quality assessment, and whether a detected variation in the activated volume can give insights on the irradiated volume. In particular, we focus on two characteristics: proton range and quantitative activation in different materials. Firstly, we evaluate how the range correctness can be checked either

N. Belcari, M.G. Bisogni, N. Camarlinghi, A. Del Guerra, M. Morrocchi, V. Rosso and G. Sportelli are with Istituto Nazionale di Fisica Nucleare, Sezione di Pisa, Italy, and with Department of Physics, University of Pisa, Italy.

A. Ferrari is with CERN, Geneva, Switzerland.

R. Kopec, D. Krzempek, K. Krzempek and P. Olko are with Institute of Nuclear Physics Polish Academy of Sciences, Krakow, Poland.

Corresponding author Valeria Rosso, email: valeria.rosso@pi.infn.it 
using separately single pencil beams or double pencil-beams. Secondly, we consider different phantoms to investigate the limits for the detectability of changes for the level of activation, and their relation to the material composition.

The study presented below can be considered as a starting point to investigate whether PET could help in image guidance procedures with selected pencil beams. The focus will be on short-time acquisitions, i.e., a few minutes after irradiation. The current study is also highly relevant for benchmarking the underlying MC models, improving the reliability of the $\mathrm{MC}$ predictions.

\section{MATERIALS AND METHODS}

\section{A. The PET system}

The DoPET detection system is based on two stationary detection heads, each one covering an area of about $16 \mathrm{~cm} \mathrm{x}$ $16 \mathrm{~cm}$ and composed by 9 independent modules. Each module consists of a 23 x 23 LYSO crystal matrix ( $2 \mathrm{~mm}$ pitch) each one coupled to an $8 \times 8$ multi-anode position sensitive photomultiplier Hamamatsu H8500. A custom designed frontend that performs the signal readout is connected to an FPGAbased data acquisition (DAQ) system. The data are recorded in list mode in a host server. Each registered event has a timestamp, allowing the analysis of different acquisition time windows [20] and the distinction of the various $\beta+$ contributors [21-24]. A more detailed description of the DoPET system can be found in [25-27].

A photo of DoPET at the Cyclotron Centre Bronowice, Institute of Nuclear Physics in (CCB IFJ PAN) in Krakow, Poland, is shown in Fig. 1. On the left is the flying case that hosts all the DAQ and the HV power supply: the compactness of all the PET system allows an ease of transport and readiness for data acquisition. A detailed description of $\mathrm{CCB}$ can be found on its official website [28].

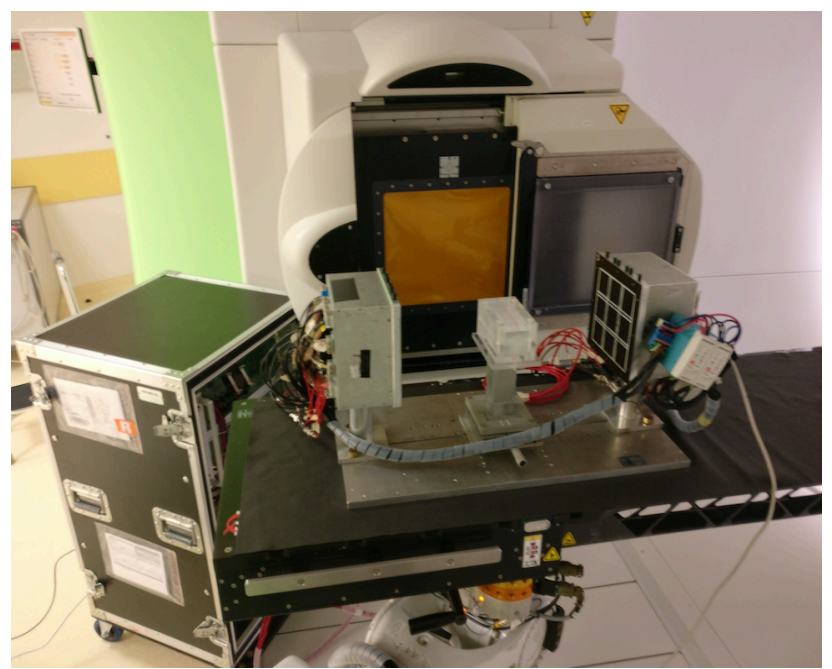

Fig. 1. Photograph of the DoPET system at CCB. The two stationary heads and the phantom are set in front of the beam nozzle, on the treatment coach. On the left the flying case containing all the DoPET data acquisition electronics and HV power supply.

\section{B. Irradiation parameters and Phantoms}

The CCB proton therapy center is a cyclotron-based facility (IBA Proteus 235), featuring an active pencil-beam scanning as beam delivery technique. The operating energy supplied from the cyclotron ranges from 70 to $230 \mathrm{MeV}$ : for more information about the commissioning of the proton scanning beam see $[29,30]$.

The DoPET system was positioned on the treatment couch, close to the gantry and the phantoms were positioned centered between the two heads, at the treatment room isocenter, as shown in Fig. 1. The distance between the PET heads was set to $48 \mathrm{~cm}$. Irradiations were performed using single or double parallel pencil-beams.

Homogeneous materials, like PMMA (Polymethyl Methacrylate), Brain equivalent tissue (hereafter referred to as BRAIN), high density polyethylene (HDPE), were used to realize homogeneous and heterogeneous phantoms. The heterogeneous ones are composed alternating slabs of PMMA and another material to get phantoms characterized by various elemental composition. Hereafter the ZEBRA-BRAIN phantom are phantoms where PMMA and BRAIN slabs alternates. Equivalently, the ZEBRA-HDPE phantom are obtained alternating HDPE slabs with PMMA. The thickness of the slabs is $2 \mathrm{~cm}$. The phantoms are parallelepiped-shaped with a transversal section of $8 \mathrm{~cm} \times 8 \mathrm{~cm}$ and a length of 14 $\mathrm{cm}$.

The elemental composition of the above-mentioned materials is well known. The relative molar fraction concentration is reported in Table I. Each phantom was considered as a whole, and the relative molar fraction concentration was calculated in terms of the two major contributors to the production of the $\beta+$ activity signals: $16-\mathrm{O}$ and 12-C.

All above phantoms entrance surface was set at about 15 $\mathrm{mm}$ along $\mathrm{z}$ : for the axis orientations see Fig. 2. The phantoms were irradiated with single pencil beams containing $10^{10}$ protons, and with a Gaussian shaped transversal profile (FWHM $\cong 10 \mathrm{~mm}$ ). The same beam currents used during the delivery of clinical treatment plans were adopted. The time necessary to deliver one pencil-beam was $4.6 \mathrm{~s}$.

Another heterogeneous phantom was prepared and divided into two sections: the upper half consists of ZEBRA-BRAIN and the lower half of PMMA. In this case, the irradiation was performed with two proton pencil-beams, aligned in the ydirection, delivering $10^{10}$ protons of $130 \mathrm{MeV}$ in each of the two sections of the phantom. The time necessary to deliver the two pencil-beams was $9.4 \mathrm{~s}: 4.6 \mathrm{~s}$ (first pencil beam) $+0.2 \mathrm{~s}$ (to move the proton pencil beam along the y direction) +4.6 s (second pencil beam). In this study were adopted pencil beams delivering $10^{10}$ protons to show the potentiality of the PET monitoring. As a reference, this protons numerosity is comparable to the number of protons per square $\mathrm{cm}$ in the last energy layer for a single-dose proton therapy treatment. 
TABLE I

RELATIVE MOLAR FRACTION CONCENTRATION

\begin{tabular}{lcc}
\hline \multicolumn{1}{c}{ Phantom } & $12-\mathrm{C}$ & $16-\mathrm{O}$ \\
\hline PMMA & & \\
BRAIN & 0.714 & 0.286 \\
$H D P E$ & 0.865 & 0.135 \\
ZEBRA-BRAIN & 1.00 & 0 \\
ZEBRA-HDPE & 0.77 & 0.23 \\
& 0.83 & 0.17
\end{tabular}

\section{PET data acquisition}

Data were acquired only after the end of the irradiations. This was due to the very high photon rate produced by the beam, which produced a partial paralysis of the photomultiplier tubes (PMTs). This condition precluded the possibility of acquiring correctly all the activity events during the irradiation and observing fast decaying isotopes. The acquisitions were performed for time intervals up to 5 minutes

Acquiring data during the irradiation at $\mathrm{CCB}$ would require: 1) reducing the PMTs gain in order to mitigate the analogue paralysis due to the limited average output current; 2) implementing timestamp acquisition for every single event (with the current system timestamp is recorded separately from events); 3) using full-beam coincidence detection techniques as the ones described in [31-33].

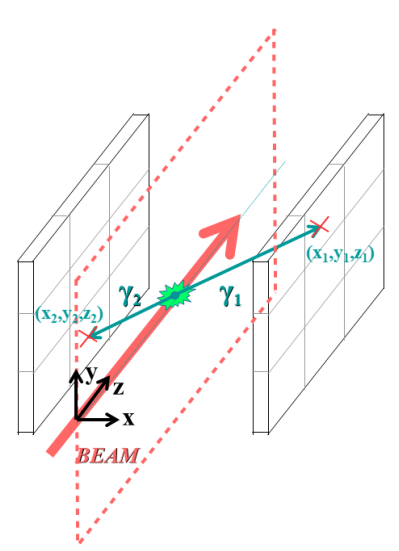

Fig. 2. Sketch of the PET system together with the used reference system. The $\mathrm{z}$ axis lays along the proton pencil beam.

\section{The Reconstruction Method}

The Straight Forward Reconstruction method (SFR) is a non-conventional reconstruction technique in PET imaging context, that allows a direct method for the calculation of the $\beta+$ activity profile along the pencil beam direction [34]. The SFR code records the annihilation points of each event, evaluating the intersection point between the line of response and the plane parallel to the DoPET heads that is passing through the beam axis ( $\mathrm{y}$ and $\mathrm{z}$ coordinates); the $\mathrm{x}$ coordinate is determined by the position of the beam axis. A sketch of the geometrical configuration behind the SFR method is reported in Fig. 2. In the same figure, the spatial reference system is also shown, where the beam direction is along the $\mathrm{z}$-axis. The spatial coordinates of the reconstructed annihilation points are stored along with the corresponding time-stamp $t$, allowing the analysis of different acquisition time windows.

\section{E. Monte Carlo simulations}

The experimental set-up geometry is reproduced with the FLUKA MC simulation framework $[35,36]$. The simulation follows the effective proton delivered from the accelerator, its interaction with the target, the production of residual nuclei, the $\beta+$ decay and tracks the positron emission and its annihilation. Finally, the two back-to-back photons produced from the annihilation are followed outside the target until they hit the detector heads $[19,37]$. The geometry of the detection system is implemented for these simulations. The position, energy deposit and time of the annihilation photons are scored event by event, every time a pair of annihilation photons leaves the target. We simulated $10^{9}$ protons for all the single pencil beam acquisitions. The characteristics of the phantom materials are reported in Table II.

The FLUKA version used for the simulations is installed on a personal computer with an Intel Core i5 4 Processor with 3.10 GHz and 3,8 GB memory running the Fedora Linux operating system. The FLUKA simulation output is directly used by the SFR reconstruction method and processed in a ROOT framework.

TABLE II

MATERIALS CHARACTERISTICS

\begin{tabular}{lccc}
\hline \hline Phantom & $\begin{array}{c}\text { Density } \\
\left(\mathrm{g} / \mathrm{cm}^{3}\right)\end{array}$ & $\begin{array}{c}\text { Z/A } \\
\text { weighted }\end{array}$ & $\begin{array}{c}\text { Average } \\
\text { excitation } \\
\text { energy }(\mathrm{eV})\end{array}$ \\
\hline PMMA & 1.18 & 0.539 & 74 \\
BRAIN & 1.05 & 0.553 & 63.5 \\
HDPE & 0.97 & 0.57 & 57.4
\end{tabular}

\section{F. Data analysis}

For each data acquisition, we derived the 1-D activity profile along the beam direction, as well as 2D images.

To find the pencil beam entrance point, the lateral activity profiles were studied.

The reconstructed activity of a selected xy plane was extracted and the profile along the y direction was calculated. Then the lateral activity profile was fitted with a Double Gaussian function to enable a more accurate evaluation of the contribution coming from the tails of the profile. The Double Gaussian function is reported in Eq. 1, where $a_{1}$ and $a_{2}$ are the amplitudes of the two Gaussians, $b$ is the centroid (location of the impinging proton beam), and $\mathrm{c}_{1}$ and $\mathrm{c}_{2}$ are related to the peak width.

$$
f(y)=a_{1} \exp -\frac{(y-b)^{2}}{c_{1}^{2}}+a_{2} \exp -\frac{(y-b)^{2}}{c_{2}^{2}}
$$

To calculate the 1D-activity-profiles a limited transversal area 
centered with respect to the proton pencil-beam was selected. As for each reconstructed activity event the SFR code records the intersection point between the line of response and the plane passing through the beam axis, the transversal selection is performed limiting the $y$ values between -7 and $+7 \mathrm{~mm}$ with respect to the beam entrance y point. From the calculated activity profile, the activity width is determined by evaluating the $50 \%$ rise and the $50 \%$ fall-off position along the activity profile (fitted with Fermi-Dirac functions, as described in [37]): this activity width is defined $\Delta \mathrm{w} 50 \%$. The data analysis focuses on the detection of the two most abundant isotopes produced during human treatment: respectively $15-\mathrm{O}$, with a half-life of $122 \mathrm{~s}$, and 11-C with a half-life of $1220 \mathrm{~s}$.

As a first check, the 1D activity profiles for homogeneous phantoms were compared with the pre-calculated profiles, simulated with FLUKA MC, in terms of $\triangle \mathrm{w} 50 \%$ (Section III A).

After that, to investigate to what extent it is possible to detect variations in phantom compositions, various phantoms were irradiated: PMMA, BRAIN, ZEBRA-BRAIN and ZEBRA-HDPE. In this case the $\Delta \mathrm{w} 50 \%$ and the total activity event number (TAEN) evaluated as the area under the profile of the experimental 1D-profiles, are calculated (Section III B).

Moreover, irradiations using two proton pencil-beams were studied to show that differences in the level of activation along the profiles can be detected and used to highlight the differences in phantom composition. Here the 1D profiles, for each pencil beam, obtained as described above, were compared with the profiles obtained with single pencil beam irradiations (Section III C).

\section{RESULTS AND DISCUSSIONS}

\section{A. Validations with homogeneous phantoms}

As a first test, two homogeneous phantoms were irradiated separately using a single $130 \mathrm{MeV}$ proton pencil-beam, and data were acquired immediately after the irradiation.

The position of the impinging pencil-beam was calculated fitting the lateral activity profile along the y direction (see Section II F). The obtained pencil-beam profile at the entrance of the phantom, evaluated at $\mathrm{z}=20 \mathrm{~mm}$, is reported in Fig. 3, together with the fitting double Gaussian function. From the fit results, the beam position resulted at $y=-0.4 \mathrm{~mm}$ : a very small shift with respect to the origin of the detection reference system (see Fig. 2) as the PET system was manually positioned.

The 1D activity profile in the two homogeneous phantoms, following the procedure described in Section II F, were calculated and, as an example, the 1D-activity profile along the z-axis, for the BRAIN phantom is reported in Fig. 4, along with the MC prediction. The $\Delta \mathrm{w} 50 \%$ were calculated and are reported in Table III, where the errors are those from the fits. The PMMA phantom activation is a consolidated result (see [23] and [37]), and constitute a reference measurement working in a proton therapy center that is new for us. Also, in this case, the $\Delta \mathrm{w} 50 \%$, experimental and MC simulations for PMMA are reported in Table III. As a reference measurement, the TAEN of the experimental 1D-profiles for PMMA, the area under the profile, was evaluated and resulted $2.8510^{5}$. To estimate the variability of the TAEN, few data taking were performed using PMMA phantoms in the same experimental conditions: the variation resulted of about $1 \%$. Variations lower than $1 \%$ for TAEN are beyond the significability of the method.

TABLE III

Activity width evaluated at 50\% (mm) and the experimental total activity event number

\begin{tabular}{lccc}
\hline \hline \multicolumn{1}{c}{ Phantom } & $\begin{array}{c}\Delta \mathrm{W} 50 \% \\
\text { experimental }\end{array}$ & $\begin{array}{c}\Delta \mathrm{w} 50 \% \\
\text { FLUKA MC }\end{array}$ & $\begin{array}{c}\text { TAEN } \\
\text { experimental }\end{array}$ \\
\hline PMMA & $100.6 \pm 0.2$ & $100.8 \pm 0.1$ & $2.85 \cdot 10^{5}$ \\
BRAIN & $110.1 \pm 0.3$ & $108.3 \pm 0.3$ & $2.10 \cdot 10^{5}$ \\
ZEBRA-BRAIN & $104.5 \pm 0.2$ & $104.2 \pm 0.3$ & $2.58 \cdot 10^{5}$ \\
ZEBRA-HDPE & $106.1 \pm 0.2$ & $105.4 \pm 0.4$ & $2.36 \cdot 10^{5}$
\end{tabular}

The agreement between the experimental profiles and the MC simulations for both homogeneous phantoms is very good either for the profile shapes and for their widths. The error values calculated with the fit procedure have sub-millimeter values (Table III). Apart from the fit error, the limit for the variation detectability of the DoPET system is $1 \mathrm{~mm}$, as was shown in a previous work [27].

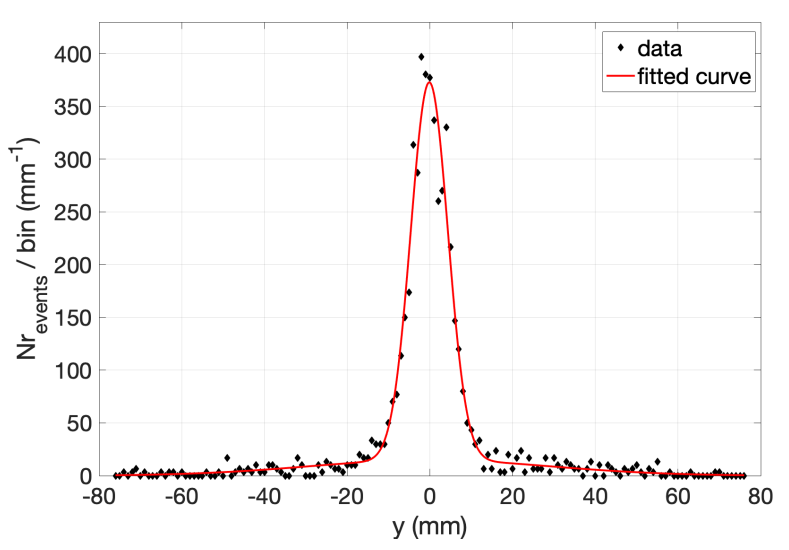

Fig. 3. Lateral proton beam profile along the y direction evaluated in PMMA for $\mathrm{z}=20 \mathrm{~mm}$. Experimental data (black diamond) together with the fit function (red line). The double Gaussian fit coefficients (with $95 \%$ confidence bounds) are: $\mathrm{a}_{1}=327.7 \pm 7.8 ; \mathrm{b}=-0.4 \pm 0.1 ; \mathrm{c}_{1}=6.24 \pm 0.2 ; \mathrm{a}_{2}$ $=13.2 \pm 4.4 ; \mathrm{c}_{2}=45.7 \pm 13.8$.

\section{B. Detectable variations of the activation level}

To investigate the variation detectability limits, heterogeneous phantoms were irradiated. Using a ZEBRABRAIN phantom, for example, the carbon content, expressed as molar fraction, was reduced from $86,5 \%$ to $77 \%$, and, at the same time, the oxygen content was increased from $13,5 \%$ to $23 \%$ with respect to the BRAIN phantom (see Table I). The 1D-activity profiles along the beam direction, reported in Fig.5, show that the activity signal reproduces the phantom 
slab structure: a lower number of activity events is present in correspondence to the BRAIN slabs with respect to the PMMA ones. Moreover, the characteristics of the BRAIN slabs reported in Table II with respect to the PMMA one, allowed a longer activation volume as shown by the measurements of the $\Delta \mathrm{w} 50 \%$, reported in Table III. A variation of about $6 \mathrm{~mm}$ between the irradiations of the two phantoms, BRAIN and ZEBRA-BRAIN, is a well detectable variation. For a more detailed comparison, the experimental profiles for these two phantoms were plotted together and are reported in Fig. 6. The level of activation in the ZEBRABRAIN and in the BRAIN phantoms is almost the same in correspondence of the BRAIN slabs, indicating that also the number of activity events can provide an indication on the local material composition. The TAEN of the experimental 1D-profiles of Fig. 6, was evaluated and resulted $2.110^{5}$ for the BRAIN phantom and $2.5810^{5}$ for the ZEBRA-BRAIN one.

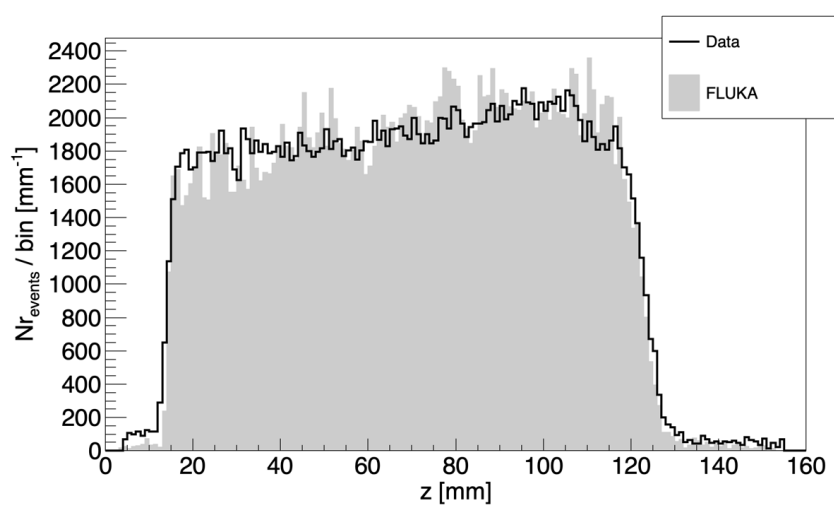

Fig. 4. 1D-activity profile for the BRAIN phantom: the experimental profile is reported as a black line along with the MC FLUKA prediction as a grey area. The area of the MC distribution was normalized to that of the experimental data. The proton beam comes from left and the phantom entrance surface is positioned at $\mathrm{z}=15 \mathrm{~mm}$.

To further investigate on the variation detectability limits, another zebra phantom was irradiated: the ZEBRA-HDPE. This heterogeneous phantom has a Carbon molar fraction close to that of the BRAIN one: $83 \%$, versus $86.5 \%$ respectively, for a variation of only $3.5 \%$ both for the Carbon, and for the Oxygen molar fraction percentages. The TAEN resulted $2.3610^{5}$, showing a further appreciable decrease. The variation in the characteristics of the BRAIN slabs and the HDPE one (see Table II) resulted in a large difference in activated volume: the corresponding variation in terms of $\Delta \mathrm{w} 50 \%$, resulted to be $4 \mathrm{~mm}$ (see Table III) between the BRAIN and the ZEBRA-HDPE phantoms.

It is also possible to compare the activation level of two ZEBRA phantoms. Fig. 7 shows the experimental 1-D profiles for the ZEBRA-BRAIN and for the ZEBRA-HDPE: a slight increase of the activated volume is visible. For the 1D profile behavior, it is visible that in correspondence to the PMMA slabs the counts are almost the same for the two phantoms, whereas a reduction is present in correspondence to the BRAIN and the HDPE slabs. The variations of the activity level, for these two materials (BRAIN and HDPE) with respect to PMMA, are related to the decrease of the Oxygen content that decreases going from PMMA to HDPE (see Table I). The MC simulation confirms these experimental results. The calculated $\Delta \mathrm{w} 50 \%$ values, reported in Table III, show that a variation of $1.6 \mathrm{~mm}$ for the experimental activity width is present. The capability to detect a variation of this order in terms of the number of used protons was investigated in a previous study. We showed that to assure an error on the determination of $\Delta \mathrm{w} 50 \%$ lower than $1.5 \mathrm{~mm}$ a number of protons greater than $210^{8}$ per pencil beam is necessary [34], a reduced proton number respect to the $10^{10}$ used in this study. The number of $10^{8}$ proton per pencil beam is comparable with the last energy layer pencil beams of a hypo-fractionated treatment plan.

This millimetric change is, in any case, a challenging variation to be detected, and the knowledge of the TAEN can reinforce the confidence in the measurement.

The percentage variation of TAEN for the two phantoms is $8.5 \%$ ((ZEBRA-BRAIN - ZEBRA-HDPE)/ ZEBRABRAIN), an easier to be detected variation. The used protons number per pencil is not a limit for the significance of the presented results. In fact, scaling back the number of used protons per pencil beam, it is possible to compare the relative sigma expressed as sigma-TAEN/TAEN. Starting from the values reported in Table III for the ZEBRA-HDPE phantom, the relative sigma varies from $2.1 \%$ for $10^{10}$ protons to $2.1 \%$ for $10^{8}$ protons, remaining a statistically significant measurement also for the less populated pencil beam.

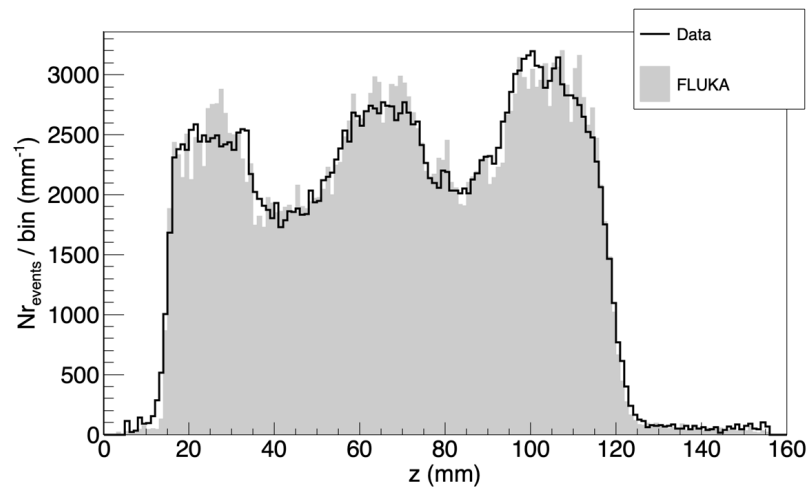

Fig. 5. 1D-activity profile for the ZEBRABRAIN phantom: the experimental profile is reported as a black line along with the MC FLUKA prediction as a gray area. The area of the MC distribution was normalized to that of the experimental data.

These measurements show how the phantom composition determines on one hand the activity width related on the phantom characteristics, and on the other hand the TAEN, related to the presence of Carbon and Oxygen in the phantom. The interaction of the proton pencil-beams with heterogeneous volumes show that pencil proton beams act as probes: in fact, the activated volume can be studied in terms of depth and activation height. The $\Delta \mathrm{w} 50 \%$ can be used as a range monitoring figure. Looking at the activation level, it may be also possible to infer, for example, information on the relative experimental content of the Oxygen in various zones 
of a volume that has to be treated. As the previous inference was based on information extracted from phantoms irradiated in various experiments, the possibility to use few pencil beams, at least two, during the same irradiation and extract the same information, has to be verified, as will be discussed next.

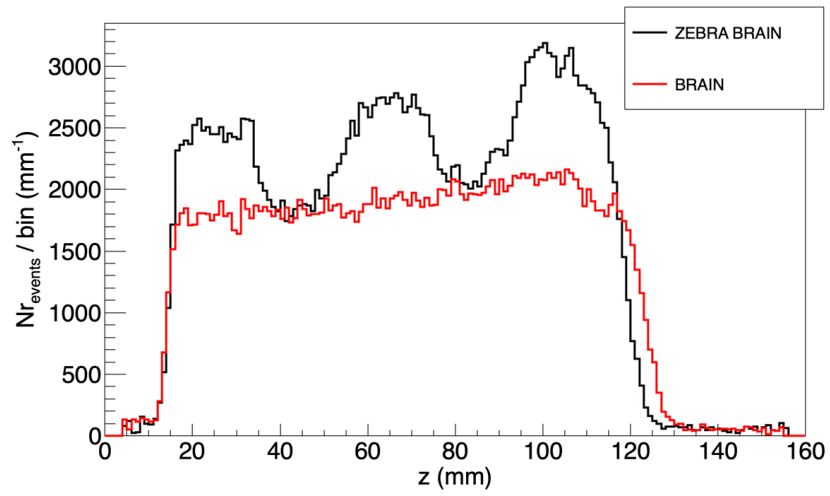

Fig. 6. 1D experimental activity profiles for the BRAIN phantom, red line and for the ZEBRABRAIN one, black line.

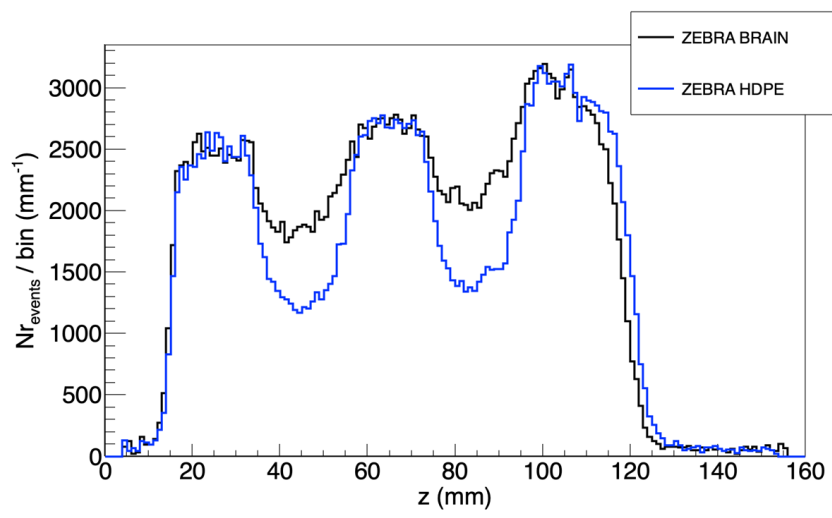

Fig. 7. 1D experimental activity profiles for the ZEBRAHDPE phantom, blue line and for the ZEBRABRAIN one, black line.

\section{Proton pencil-beams as probes}

The phantom divided into two sections, where the upper half is ZEBRA-BRAIN and the lower half is PMMA, was irradiated. To check the two pencil-beams delivery position, the activity profile along the y direction was studied, as done for the PMMA phantom. From the fit evaluation, the lower pencil-beam was found to be centered at $y=-9 \mathrm{~mm}$, whereas the upper one was centered at $y=15.3 \mathrm{~mm}$. The 2D-image of the activity signal in the yz-plane reported in Fig. 8, shows clearly two activated volumes corresponding to the two delivered pencil-beams. Different activity widths and signal heights are well distinguishable in the 2D-image, but to better appreciate the variations, the two $1 \mathrm{D}$-activity profiles in correspondence to the two activated volumes, were calculated and are reported in Fig. 9. The two activity widths are in good agreement (within $1 \mathrm{~mm}$ ) with the corresponding activity widths obtained with single pencil-beams irradiations. Nevertheless, we observe a difference in the level of activation along the profiles. In fact, the PMMA slabs do not have the same activation level of the homogeneous section as expected, and also the TAENs are different with respect to the single-pencils irradiation case. These differences have two origins: a different geometrical irradiation point $(y=-0.4$ for the single pencil irradiation case, whereas $\mathrm{y}=-9 \mathrm{~mm}$ and $\mathrm{y}=$ $15.3 \mathrm{~mm}$ for the two pencils irradiation case) and a different beam delivery modality. For the case of two pencils irradiation, the time window for the reconstruction of the two activated volumes started jointly shortly after the delivery of the second pencil-beam, creating an asymmetry between the detected activity events of the two pencils.

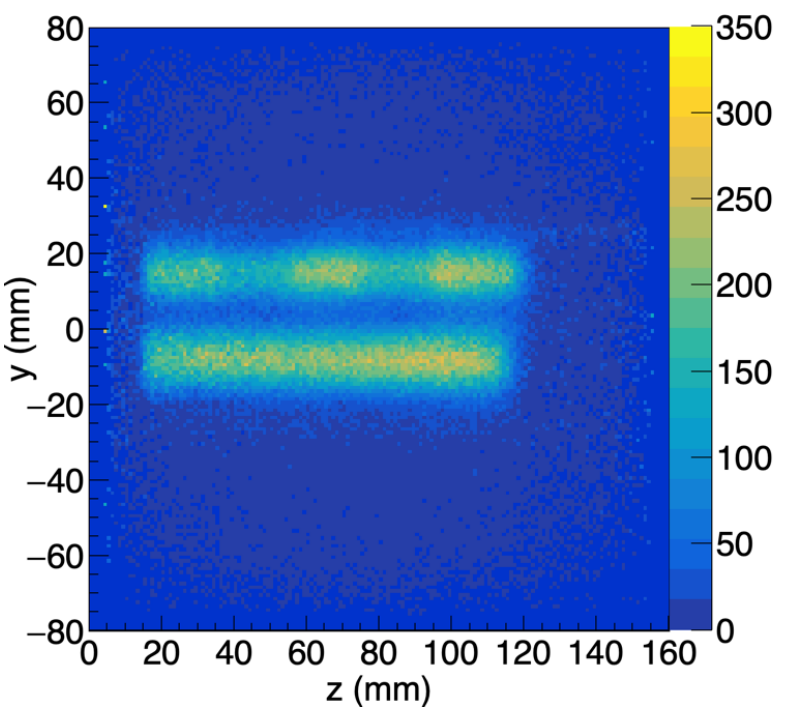

Fig. 8. 2D activity distribution in yz plane of the activated volume: the lower part corresponds to the homogeneous PMMA irradiation, whereas the upper part corresponds to ZEBRA-BRAIN section irradiation.

To take into account these differences in space and time, FLUKA simulations were performed. Two simulations, one for the pencil-beam impinging in $\mathrm{y}=-9 \mathrm{~mm}$ and the other in $\mathrm{y}=15.3 \mathrm{~mm}$, irradiating always a PMMA phantoms, were performed and the results compared to the single pencil-beam case, where $\mathrm{y}$ was $\mathrm{y}=-0.4$. A space-coefficient was calculated so that TAEN for the two pencil-beam irradiation case was each one the same with respect to the single pencilbeam irradiation case. These two space-coefficient are then corrected for the difference due to the variation in the time protons delivery. For this time correction, again the PMMA MC simulation was considered. The TAEN was calculated for two time windows, both of $300 \mathrm{~s}$, starting at different times: the first one, the already used one, that starts $4.6 \mathrm{~s}$ after the beginning of the irradiation, and a second one that starts $9.4 \mathrm{~s}$ after the beginning of the irradiation. The coefficient that equalized these two different TAENs was calculated and applied, multiplicatively, to the previously calculated spacecoefficients. The final coefficient for the pencil beam centered in $\mathrm{y}=-9 \mathrm{~mm}$ resulted to be 1.15 whereas the coefficient for $\mathrm{y}$ $=15.3 \mathrm{~mm}$ was 1.30 . These coefficients were applied to the 1 - 
D profiles reported in Fig. 9 and the result is reported in Fig. 10 along with the corresponding 1-D profiles obtained for two single pencil-beam irradiation cases. The two couples of profiles overly well either in terms of $\Delta \mathrm{w} 50 \%$, in the profiles behavior, and also the TAEN values are in good agreement. In fact, for the PMMA section the 2-pencil TAEN resulted to be $2.8310^{5}$ whereas its value for the single irradiation case was $2.8510^{5}$, for a percentage variation smaller than $1 \%$. Correspondently for the ZEBRA-BRAIN section, the 2-pencil TAEN resulted $2.6710^{5}$, whereas for the single irradiation case TAEN was $2.5810^{5}$ : in this case the percentage variation is $3.5 \%$. This last evaluation is an indication for the limit of the capability to detect variations in the TAEN.

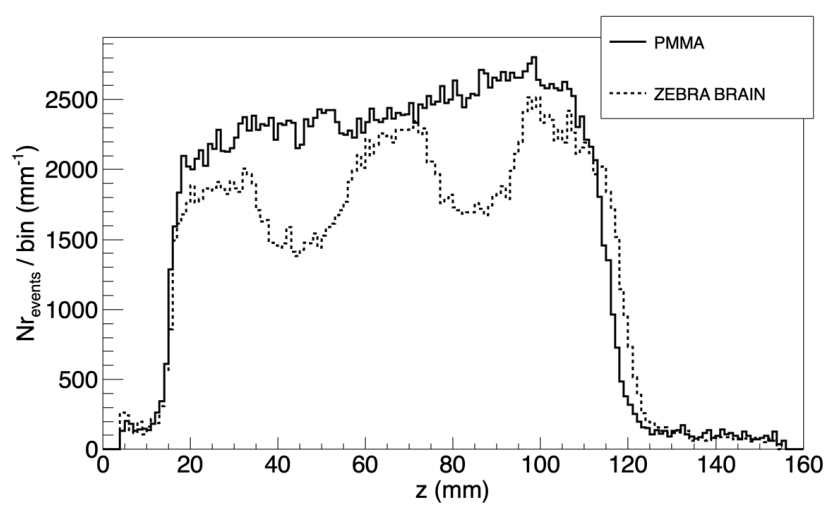

Fig. 9. 1D experimental activity profiles for the PMMA section, black line, and for the ZEBRABRAIN section, dotted grey line. Two proton pencil beams, sent consecutively, generate the activated volumes.

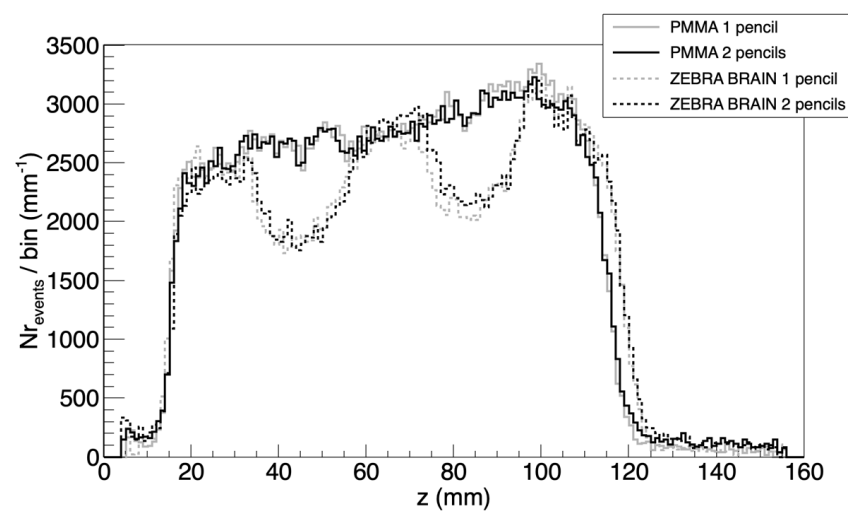

Fig. 10. Comparison between the 1D experimental activity profiles obtained sending two proton pencil beams consecutively, black lines, and two proton pencil beams separately, grey lines. The 1D experimental activity profiles for the PMMA section are reported as continuous lines, whereas for the ZEBRABRAIN section, dotted lines are adopted.

\section{CONCLUSIONS}

In this work we showed a potential tool for the treatment quality assessment in proton therapy based on an in-beam PET system, DoPET, along with FLUKA MC simulations. The technique was tested on phantoms comparing the precalculated and the measured activity distributions. Data acquisitions were performed after the treatment delivery and lasted about 5 minutes, increasing, by a small amount, the overall treatment room occupancy.

The data acquisitions were performed immediately after the irradiations due to the DoPET features: the overcome of these limitations requires major updates that are left for future works.

The results using two proton pencil-beams as probes to test heterogeneous volumes were as expected: the phantoms compositions of the irradiated volumes are expressed through the change of the $\Delta w 50 \%$ and the total activity event number.

In view of hypo-fractionated treatments, a range check with a few selected pencil beams, will provide a sufficiently precise determination of $\Delta \mathrm{w} 50 \%$ and TAEN.

The number of proton pencil-beams adopted in this study was limited to two, but this number can be increased, taking into account that in any case the activated volumes produced by the pencil beams have to not greatly overlap each other. This kind of check could represent a starting point for image guided hadrontherapy using PET in case hypo-fractionated treatment is applied.

These studies could be also a starting point in using PET for adaptive strategies in proton therapy.

\section{REFERENCES}

[1] F. Tommasino and M. Durante, "Proton radiobiology", Cancers, 2015, vol. 7, pp. 353-38. DOI: 10.3390/cancers 7010353

[2] A. Knopf and A. Lomax, "In vivo proton range verification: A review", Physics in Medicine and Biology, 2013, vol. 58, no. 15, R131. DOI: 10.1088/0031-9155/58/15/R131

[3] K. Parodi, W. Enghardt, T. Haberer, "In-beam PET measurements of beta+ radioactivity induced by proton beams", Phys. Med. Biol. 2002, vol. 47, 21-36. DOI: 10.1088/0031-9155/47/1/302

[4] Y. Iseki, T. Kanai, M. Kanazawa, A. Kitagawa et al., "Range verification system using positron emitting beams for heavy-ion radiotherapy", Phys Med Biol, 2004, vol. 49 (14):3179-95. DOI: 10.1088/0031-9155/49/14/012

[5] T. Nishio, A. Miyatake, T. Ogino, K. Nakagawa, N. Saijo, H. Esumi, "The development and clinical use of a beam ON-LINE PET system mounted on a rotating gantry port in proton therapy", Int J Radiat Oncol Biol Phys, 2010, vol. 76 (1): 277-86. DOI: 10.1016/j.ijrobp.2009.05.065

[6] H. Tashima, T. Yamaya, E. Yoshida, S. Kinouchi, M. Watanabe, E. Tanaka, "A single-ring OpenPET enabling PET imaging during radiotherapy”, Phys Med Biol, 2012, vol. 57 (14): 4705-18. DOI: 10.1088/0031-9155/57/14/4705

[7] G. Sportelli et al., "First full-beam PET acquisitions in proton therapy with a modular dual-head dedicated system", Phys Med Biol, 2014, vol. 59 (1): 43-60, DOI: 10.1088/0031-9155/59/1/43

[8] Y. Shao, X. Sun, K. Lou, X.R. Zhu, D. Mirkovich et al. "In-beam PET imaging for on-line adaptive proton therapy: an initial phantom study", Phys Med Biol, 2014, vol. 59 (14): 3373-88. DOI: $10.1088 / 0031-9155 / 59 / 13 / 3373$

[9] X. Zhu and G. El Fakhri, "Proton Therapy Verification with PET Imaging", Theranostics, 2013, vol. 3 (10), 731-740. DOI: $10.7150 /$ thno. 5162

[10] M.G. Bisogni et al., "INSIDE in-beam Positron Emission Tomography system for particle range monitoring in hadrontherapy, J. Med. Imag. Vol. 4, 2017, 011005 DOI: 10.1117/1.JMI.4.1.011005

[11] P. Crespo et al., "Direct time-of-flight for quantitative, real-time inbeam PET: A concept and feasibility study" Phys. Med. Biol., 
2007, vol. 52 (23), pp. 6795-6811. DOI:

$10.1088 / 0031-9155 / 52 / 23 / 002$

[12] S. Binet et al., "Construction and First Tests of an in-beam PET Demonstrator Dedicated to the Ballistic Control of Hadrontherapy Treatments With $65 \mathrm{MeV}$ Protons" IEEE Trans Rad Plasma Med Sciences, 2018, vol. 2 (1), 51-60. DOI: 10.1109/TRPMS.2017.2780447

[13] E Yoshida et al., "Development of a Whole-Body Dual Ring OpenPET for in-Beam PET" IEEE Trans Rad Plasma Med Sciences, 2017, vol. 1 (4), 293-300. DOI: 10.1109/TRPMS.2017.2703823

[14] V. Ferrero et al., "Double-Field Hadrontherapy Treatment Monitoring with the INSIDE In-Beam PET Scanner: Proof of Concept”, IEEE Trans Rad Plasma Med Sciences, 2018, vol. 2 (6). 588-593. DOI: $10.1109 /$ TRPMS.2018.2870478

[15] V. Ferrero et al., "Online proton therapy monitoring: clinical test of a Silicon-photodetector based in-beam PET", Scientific Reports, 2018, vol. 8, 4100, DOI: 10.1038/s41598-018-22325-6

[16] J. Handracket al., "Sensitivity of post treatment positron emission tomography/computed tomography to detect inter-fractional range variations in scanned ion beam therapy", ACTA ONCOLOGICA, 2017, vol. 56 (11), 1451-1458, DOI: 10.1080/0284186X.2017.1348628

[17] C. Hee et al., "Clinical Application of In-Room Positron Emission Tomography for In Vivo Treatment Monitoring in Proton Radiation Therapy", International Journal of Radiation Oncology*Biology*Physics, 2013, vol. 86 (1), 183-189, DOI: 10.1016/j.ijrobp.2012.12.010

[18] X. Zhu et al., "Monitoring proton radiation therapy with in-room PET imaging", Phys Med Biol, 2011, vol. 56: 4041-57, DOI: 10.1088/00319155/56/13/019

[19] A. C. Kraan et al., "Proton range monitoring with in-beam PET: Monte Carlo activity predictions and comparison with cyclotron data", Physica Medica, 2014, vol. 30.5, pp. 559-569, DOI: 10.1016/j.ejmp.2014.04.003

[20] A. Topi et al., "In-beam PET monitoring technique for proton therapy: experimental data and Monte Carlo prediction", RAD Conference Proceedings, 2018, vol. 3, 149-153, DOI: 10.21175/RadProc. 2018.32

[21] F. Attanasi, et al., "Characterization of an in-beam PET prototype for proton therapy with different target compositions", IEEE Trans Nucl Sci, 2010, vol. 57(3), pp. 1563-1569, DOI: 10.1109/TNS.2010.2048124

[22] K. Grogg et al., "Mapping 15-O production rate for proton therapy verification", Int J Radiat Oncol Biol Phys, 2015, 92(2):453-59, DOI: 10.1016/j.ijrobp.2015.01.023

[23] P. Cambraia Lopes et al., "First in situ TOF-PET study using digital photon counters for proton range verification", Phys Med Biol, 2016, 61: 6203-6230. DOI: 10.1088/0031-9155/61/16/6203

[24] A. C. Kraan et al., "Analysis of time-profiles with in-beam PET monitoring in charged particle therapy", Journal of Instrumentation, 2019, Vol. 14 (2) pp. C02001. DOI: 10.1088/1748-0221/14/02/C02001

[25] S. Vecchio et al., "A PET prototype for in-beam monitoring of proton therapy" IEEE Trans Nucl Sci, 2009, 56 51-56

[26] E. Fabbiani et al., "Performance evaluation of a LYSO-based PET scanner for monitoring of dose delivery in hadrontherapy", Journal of Instrumentation, 10, C12029, 2015, 1-11. DOI:10.1088/17480221/10/12/C12029
[27] V. Rosso et al., "DoPET an in-treatment monitoring system for proton therapy at $62 \mathrm{MeV}$ ", Journal of Instrumentation, 2016 vol. 11, p.1-8 C12029, DOI: $10.1088 / 1748-0221 / 11 / 12 / C 12029$

[28] CCB. (last accessed online November 2018). Cyclotron Centre Bronowice, [Online]. Available:

https://ccb.ifj.edu.pl/en.home.html

[29] J. Kunst et al., "Mailed dosimetric audit of therapeutic proton beams using thermoluminescence MTS-N (LiF:Mg,Ti) powder first results", Radiation Measurements, 2017, vol. 106, pp. 312314. DOI: $10.1016 /$ j.radmeas.2017.03.031

[30] M. Liszka et al, "Ion recombination and polarity correction factors for a plane-parallel ionization chamber in a proton scanning beam”, Medical Physics, 2017, vol. 45, 1. DOI: 10.1002/mp. 12668

[31] P. Crespo et al., "Suppression of random coincidences during inbeam PET measurements at ion beam radiotherapy facilities", IEEE Trans Nucl Sci, 2005, vol. 52 (4), 980-987. DOI: 10.1109/TNS.2005.852637

[32] M.A. Piliero et al., "Full-beam performances of a PET detector with synchrotron therapeutic proton beams", Phys Med Biol, 2016, Vol. 61 (23), N650-N666 DOI: 10.1088/0031-9155/61/23/N650

[33] E. Kostara et al., "Particle beam microstructure reconstruction and coincidence discrimination in PET monitoring for hadron therapy", Phys Med Biol, 2019, Vol. 64 (3), 035001, DOI: 10.1088/1361-6560/aafa28

[34] S. Muraro et al., "Low statistics activity reconstruction methods with the DoPET system", Nuclear Physics, 2018, to be published, DOI: 10.1016/j.nima.2018.10.015

[35] G. Battistoni et al., "The FLUKA Code: An Accurate Simulation Tool for Particle Therapy", Frontiers in Oncology, 2016, vol. 6, p. 116. ISSN: 2234-943X, DOI: 10.3389/fonc.2016.00116

[36] R.S. Augusto et al., "An overview of recent developments in FLUKA PET tools”, Physica Medica, 2018, vol. 54, pp. 189-199, DOI: $10.1016 /$ j.ejmp.2018.06.636

[37] S. Muraro et al., "Proton therapy treatment monitoring with the DoPET system: Activity range, positron emitters evaluation and comparison with Monte Carlo predictions", Journal of Instrumentation, 2017, vol. 12, C12026. DOI: $10.1088 / 17480221 / 12 / 12 / \mathrm{C} 12026$ 